\title{
Stably co-tame polynomial automorphisms over commutative rings
}

\author{
Shigeru Kuroda*
}

\begin{abstract}
We say that a polynomial automorphism $\phi$ in $n$ variables is stably co-tame if the tame subgroup in $n$ variables is contained in the subgroup generated by $\phi$ and affine automorphisms in $n+1$ variables. In this paper, we give conditions for stably co-tameness of polynomial automorphisms.
\end{abstract}

\section{Introduction}

Let $R$ be a commutative ring of characteristic $p \neq 1, R[\mathbf{x}]:=R\left[x_{1}, \ldots, x_{n}\right]$ the polynomial ring in $n$ variables over $R$, and $\mathrm{GA}_{n}(R):=\operatorname{Aut}_{R} R[\mathbf{x}]$ the automorphism group of the $R$-algebra $R[\mathbf{x}]$. We identify each $\phi \in \operatorname{GA}_{n}(R)$ with the $n$-tuple $\left(\phi\left(x_{1}\right), \ldots, \phi\left(x_{n}\right)\right)$ of elements of $R[\mathbf{x}]$. The composition is defined by

$$
\phi \circ \psi=\left(g_{1}\left(f_{1}, \ldots, f_{n}\right), \ldots, g_{n}\left(f_{1}, \ldots, f_{n}\right)\right)
$$

for $\phi=\left(f_{1}, \ldots, f_{n}\right), \psi=\left(g_{1}, \ldots, g_{n}\right) \in R[\mathbf{x}]^{n}$. For each $r \geq 1$, we regard $\mathrm{GA}_{n}(R)$ as a subgroup of $\mathrm{GA}_{n+r}(R)$ by identifying each $\phi \in \mathrm{GA}_{n}(R)$ with the unique extension $\tilde{\phi} \in \mathrm{GA}_{n+r}(R)$ of $\phi$ defined by $\tilde{\phi}\left(x_{n+i}\right)=x_{n+i}$ for $i=1, \ldots, r$.

We say that $\phi \in \mathrm{GA}_{n}(R)$ is affine if $\phi=\left(x_{1}, \ldots, x_{n}\right) A+b$ for some $A \in G L_{n}(R)$ and $b \in R^{n}$, and set $\operatorname{Aff}_{n}(R):=\left\{\phi \in \mathrm{GA}_{n}(R) \mid \phi\right.$ is affine $\}$. We define

$$
\epsilon(f):=\left(x_{1}+f, x_{2}, \ldots, x_{n}\right) \in \mathrm{GA}_{n}(R) \text { for each } f \in R[\hat{\mathbf{x}}]:=R\left[x_{2}, \ldots, x_{n}\right],
$$

and set $\mathrm{E}_{n}(R):=\{\epsilon(f) \mid f \in R[\hat{\mathbf{x}}]\}$. We call $\mathrm{T}_{n}(R):=\left\langle\operatorname{Aff}_{n}(R), \mathrm{E}_{n}(R)\right\rangle$ the tame subgroup, and elements of $\mathrm{T}_{n}(R)$ are said to be tame. Here,

*Partly supported by JSPS KAKENHI Grant Number 15 K04826 
for subsets $S_{1}, \ldots, S_{r}$ and elements $g_{1}, \ldots, g_{s}$ of a group $G$, we denote by $\left\langle S_{1}, \ldots, S_{r}, g_{1}, \ldots, g_{s}\right\rangle$ the subgroup of $G$ generated by $\bigcup_{i=1}^{r} S_{i} \cup\left\{g_{1}, \ldots, g_{s}\right\}$.

If $n \geq 3$ and $R$ contains $\mathbf{Q}$, then $\mathrm{T}_{n}(R)=\left\langle\operatorname{Aff}_{n}(R), \sigma\right\rangle$ holds for $\sigma=\epsilon\left(x_{2}^{2}\right)$ by Derksen (cf. [Es, Thm. 5.2.1]). We remark that Derksen's theorem requires that $R$ is generated by $R^{*}$ as a $\mathbf{Q}$-vector space, but this assumption is in fact unnecessary (cf. \$3). When $R$ is a field and $p=0$, Bodnarchuk $\mathrm{B}$ proved a similar result for more general $\sigma$ 's. The situation is different if $p$ is a prime. Maubach-Willems [MW] showed that $\mathrm{T}_{3}\left(\mathbf{F}_{2}\right) \neq\left\langle\mathrm{Aff}_{3}\left(\mathbf{F}_{2}\right), \epsilon\left(x_{2}^{2}\right)\right\rangle$, and conjectured that, if $n \geq 3$ and $R$ is a finite field, then no finite subset $E$ of $\mathrm{T}_{n}(R)$ satisfies $\mathrm{T}_{n}(R)=\left\langle\operatorname{Aff}_{n}(R), E\right\rangle$.

Edo [Ed] found a class of $\phi \in \mathrm{GA}_{n}(R)$ for which $\left\langle\operatorname{Aff}_{n}(R), \phi\right\rangle$ contains $\mathrm{T}_{n}(R)$. Such $\phi$ is said to be co-tame. If $R$ is a field, no element of $\operatorname{GA}_{2}(R)$ is co-tame thanks to Jung [J] and van der Kulk [K]. For $n \geq 3$, it is easy to find elements of $\mathrm{GA}_{n}(R) \backslash \operatorname{Aff}_{n}(R)$ which are not co-tame if $R$ is not a field (cf. (4.1)), or if $p$ is a prime (cf. [EK, $\S 1$ (d)]). In the case where $n \geq 3, R$ is a field and $p=0$, the first example of such an automorphism was found by Edo-Lewis [EL] for $n=3$.

Recall that $\phi \in \mathrm{GA}_{n}(R)$ is said to be stably tame if $\phi$ belongs to $\mathrm{T}_{n+1}(R)$. It is known that some non-tame automorphisms are stably tame (cf. [BEW], $[\mathrm{N}],[\mathrm{SU}],[\mathrm{S}]$ ). The following is an analogue to the stably tame automorphisms.

Definition 1.1. We say that $\phi \in \mathrm{GA}_{n}(R)$ is stably co-tame if $\left\langle\operatorname{Aff}_{n+1}(R), \phi\right\rangle$ contains $\mathrm{T}_{n}(R)$, or equivalently $\left\langle\operatorname{Aff}_{n+1}(R), \phi\right\rangle$ contains $\mathrm{E}_{n}(R)$.

Clearly, co-tame automorphisms are stably co-tame. When $R$ is a field, there exist elements of $\mathrm{T}_{3}(R)$ which are not co-tame but stably co-tame in both cases $p=0$ and $p>0$ (cf. 86,1). The purpose of this paper is to study when elements of $\mathrm{GA}_{n}(R)$ are stably co-tame or not. If $R$ contains an infinite field, we have a necessary and sufficient condition for stably cotameness (Corollary 2.4).

This paper is organized as follows. The main results are stated in Section 2, and three key results are proved in Sections 3, 4 and 5, In Section 6, we study stably co-tameness of the example of Edo-Lewis. We also discuss a technique which is useful when $R$ does not contain an infinite field.

\section{Main results}

Since $\mathrm{T}_{1}(R)=\operatorname{Aff}_{1}(R)$, we always assume that $n \geq 2$ unless otherwise stated. Take any $\phi \in \mathrm{GA}_{n}(R)$. We define $M_{\phi}$ to be the $R$-submodule of 
$R[\mathbf{x}]$ generated by $1, x_{i}$ and $\eta\left(\phi\left(x_{i}\right)\right)$ for $i=1, \ldots, n$ and $\eta \in \operatorname{Aff}_{n}(R)$. By definition, we have

$$
\eta\left(M_{\phi}\right) \subset M_{\phi} \quad \text { for each } \eta \in \operatorname{Aff}_{n}(R) .
$$

The following theorem holds for any commutative ring $R$ of characteristic $p \neq 1$.

Theorem 2.1. $\phi \in \mathrm{GA}_{n}(R)$ is stably co-tame in the following four cases:

(a) $M_{\phi}$ contains $x_{i} x_{j}$ for some $1 \leq i<j \leq n$.

(b) 2 is a unit of $R$, and $M_{\phi}$ contains $x_{i}^{2}$ for some $1 \leq i \leq n$.

(c) $n=p=2$ and $M_{\phi}$ contains $x_{1}^{2} x_{2}$ or $x_{1} x_{2}^{2}$.

(d) $n=p=2, M_{\phi}$ contains $x_{1}^{3}$ or $x_{2}^{3}$, and there exists $\xi \in R^{*}$ satisfying $\xi+1 \in R^{*}$.

Next, assume that $p$ is zero or a prime. For $a \in R$ and $t_{1}, \ldots, t_{n} \geq 0$, we call $a x_{1}^{t_{1}} \cdots x_{n}^{t_{n}}$ a good monomial in the following five cases:

(I) $p=0$ and $t_{1}+\cdots+t_{n} \geq 2$.

(II) $p \geq 2$ and there exist $1 \leq i<j \leq n$ such that $t_{i}, t_{j} \equiv 1(\bmod p)$.

(III) $p \geq 3$ and there exists $1 \leq i \leq n$ such that $t_{i} \not \equiv 0,1(\bmod p)$.

(IV) $n=p=2$ and there exist $i, j \in\{1,2\}$ such that $t_{i} \equiv 1$ and $t_{j} \equiv 2$ $(\bmod 4)$.

(V) $n=p=2$ and there exists $1 \leq i \leq 2$ such that $t_{i} \equiv 3(\bmod 4)$.

For each $f \in R[\mathbf{x}]$, let $C_{f}$ denote the set of the coefficients of good monomials appearing in $f$. We define $I_{\phi}$ to be the ideal of $R$ generated by $\bigcup_{i=1}^{n} C_{\phi\left(x_{i}\right)}$.

Theorem 2.2. Assume that $p$ is zero or a prime. If $\phi \in \operatorname{GA}_{n}(R)$ satisfies $I_{\phi} \neq R$, then $\phi$ is not stably co-tame.

Throughout this paper, let $k$ be a field. When $R$ is a commutative $k$ algebra, we say that $f \in R[\mathbf{x}]$ satisfies the degree condition if

$$
\operatorname{deg}_{x_{i}}^{s} f \leq \# k-2 \text { for } i=1, \ldots, n \text {. }
$$

Here, $\operatorname{deg}_{x_{i}}^{s} f$ denotes the separable degree of $f$ if $p>0$, and the standard degree $\operatorname{deg}_{x_{i}} f$ of $f$ if $p=0$, as a polynomial in $x_{i}$. The separable degree of a polynomial $g(x) \in R[x]$ in one variable is defined as the degree of $h(x) \in$ $R[x] \backslash R\left[x^{p}\right]$ such that $h\left(x^{p^{e}}\right)=g(x)$ for some $e \geq 0$. We say that $\phi \in$ $\mathrm{GA}_{n}(R)$ satisfies the degree condition if $\phi\left(x_{i}\right)$ satisfies the degree condition for $i=1, \ldots, n$.

Now, we define $J_{\phi}$ to be the ideal of $R$ generated by the union of $C_{f}$ for $f \in \sum_{i=1}^{n} R \phi\left(x_{i}\right)+\sum_{i=1}^{n} R x_{i}$ satisfying the degree condition. Since no good 
monomial is linear, $J_{\phi}$ is contained in $I_{\phi}$. If $\phi$ satisfies the degree condition, then $J_{\phi}$ is equal to $I_{\phi}$. Hence, (ii) of the following theorem is a consequence of (i).

Theorem 2.3. Let $k$ be a field, $R$ a commutative $k$-algebra, and $\phi \in \mathrm{GA}_{n}(R)$.

(i) If $J_{\phi}=R$, then $\phi$ is stably co-tame.

(ii) If $\phi$ satisfies the degree condition and if $I_{\phi}=R$, then $\phi$ is stably co-tame.

From Theorems 2.2 and 2.3 (ii), we obtain the following corollary.

Corollary 2.4. Let $k$ be an infinite field, and $R$ a commutative $k$-algebra. Then, $\phi \in \mathrm{GA}_{n}(R)$ is stably co-tame if and only if $I_{\phi}=R$.

In particular, if $R=k$ is an infinite field, then $\phi \in \mathrm{GA}_{n}(k)$ is stably co-tame if and only if a good monomial appears in $\phi\left(x_{i}\right)$ for some $i$. When $p=0$, this is the same as $\phi$ is non-affine.

In the following three sections, we prove Theorems 2.1, 2.2 and 2.3 (i).

\section{Proof of Theorem 2.1}

Let $R$ be any commutative ring, and $\Gamma$ a subgroup of $\operatorname{GA}_{n+1}(R)$ containing $\operatorname{Aff}_{n+1}(R)$, where $n \geq 2$. First, we study properties of $\Gamma$. Define

$$
\hat{\epsilon}(f):=\left(x_{1}, \ldots, x_{n}, x_{n+1}+f\right) \in \mathrm{GA}_{n+1}(R) \text { for each } f \in R[\mathbf{x}] .
$$

We identify each permutation $\sigma \in S_{n+1}$ with $\left(x_{\sigma(1)}, \ldots, x_{\sigma(n+1)}\right) \in \operatorname{Aff}_{n+1}(R)$, and write $\phi^{\sigma}:=\sigma^{-1} \circ \phi \circ \sigma$ for $\phi \in \mathrm{GA}_{n+1}(R)$. Then, we have the following: (A) If $\phi=\left(f_{1}, \ldots, f_{n}\right) \in \mathrm{GA}_{n}(R)$ belongs to $\Gamma$, then $\hat{\epsilon}\left(f_{i}\right)=\phi \circ \hat{\epsilon}\left(x_{i}\right) \circ \phi^{-1}$ belongs to $\Gamma$ for each $1 \leq i \leq n$.

(B) If $f, g \in R[\hat{\mathbf{x}}]$ satisfy $\epsilon(f), \epsilon\left(g x_{n+1}\right) \in \Gamma$, then we have $\hat{\epsilon}(f)=\epsilon(f)^{(1, n+1)} \in$ $\Gamma$, and so

$$
\epsilon(f g)=\hat{\epsilon}(f) \circ \epsilon\left(g x_{n+1}\right) \circ \hat{\epsilon}(f)^{-1} \circ \epsilon\left(g x_{n+1}\right)^{-1} \in \Gamma .
$$

In particular, $\epsilon(f) \in \Gamma$ implies $\epsilon(a f) \in \Gamma$ for each $f \in R[\hat{\mathbf{x}}]$ and $a \in R$, since $\epsilon\left(a x_{n+1}\right)$ belongs to $\operatorname{Aff}_{n+1}(R)$, and hence to $\Gamma$.

When $\mathrm{Q} \subset R$ and $n \geq 3$, Derksen showed that $\mathrm{T}_{n}(R)$ is generated by $\operatorname{Aff}_{n}(R)$ and $\left\{\epsilon\left(a x_{2}^{2}\right) \mid a \in R\right\}$, and $\mathrm{T}_{n}(R)=\left\langle\operatorname{Aff}_{n}(R), \epsilon\left(x_{2}^{2}\right)\right\rangle$ holds if $R$ is generated by $R^{*}$ as a $\mathbf{Q}$-vector space (cf. [ES, Thm. 5.2.1]). We remark that, since $n \geq 3$, the first statement and (B) imply that $\mathrm{T}_{n}(R)=\left\langle\operatorname{Aff}_{n}(R), \epsilon\left(x_{2}^{2}\right)\right\rangle$ whenever $\mathrm{Q} \subset R$. 
Lemma 3.1. If $\Gamma$ contains $\hat{\epsilon}\left(x_{1} x_{2}\right)$, or if $n=2$ and $\Gamma$ contains $\hat{\epsilon}\left(x_{1} x_{2}^{2}\right)$, then $\Gamma$ contains $\mathrm{T}_{n}(R)$.

Proof. We show that $\Gamma$ contains $\epsilon(f)$ for each $f \in R[\hat{\mathbf{x}}]$. Since $\epsilon(g+h)=$ $\epsilon(g) \circ \epsilon(h)$ holds for each $g, h \in R[\hat{\mathbf{x}}]$, we may assume that $f$ is a monomial. In the former case, $\Gamma$ contains $\hat{\epsilon}\left(x_{1} x_{2}\right)^{(1, n+1)(2, i)}=\epsilon\left(x_{i} x_{n+1}\right)$ for each $2 \leq i \leq n$. By (B), it follows that $\epsilon(f) \in \Gamma$ implies $\epsilon\left(x_{i} f\right) \in \Gamma$. Since $\Gamma$ contains $\epsilon(a)$ for each $a \in R$, the assertion follows by induction on $\operatorname{deg} f$. In the latter case, $\Gamma$ contains $\hat{\epsilon}\left(x_{1} x_{2}^{2}\right)^{(1,3)}=\epsilon\left(x_{2}^{2} x_{3}\right)$. Hence, $\epsilon\left(a x_{2}^{l}\right) \in \Gamma$ implies $\epsilon\left(a x_{2}^{l+2}\right) \in \Gamma$ for each $a \in R$ and $l \geq 0$ similarly. Since $\Gamma$ contains $\epsilon(a)$ and $\epsilon\left(a x_{2}\right)$, it follows that $\Gamma$ contains $\epsilon\left(a x_{2}^{l}\right)$ for all $l \geq 0$.

The following two implications hold for the conditions listed in Theorem 2.1,

Lemma 3.2. (b) implies (a), and (d) implies (c).

Proof. By (2.1), (b) implies $\left(x_{1}+x_{2}\right)\left(x_{1}-x_{2}\right)=x_{1}^{2}-x_{2}^{2} \in M_{\phi}$. Since 2 is a unit of $R$, we have $\left(x_{1}+x_{2}, x_{1}-x_{2}, x_{3}, \ldots, x_{n}\right) \in \operatorname{Aff}_{n}(R)$. Hence, $M_{\phi}$ contains $x_{1} x_{2}$ by (2.1). Similarly, (d) implies $x_{1}^{2} x_{2}+x_{1} x_{2}^{2}=x_{1}^{3}+x_{2}^{3}+\left(x_{1}+x_{2}\right)^{3} \in M_{\phi}$. Hence, $M_{\phi}$ contains $\left(\left(\xi x_{1}\right)^{2} x_{2}+\xi x_{1} x_{2}^{2}\right)+\xi^{2}\left(x_{1}^{2} x_{2}+x_{1} x_{2}^{2}\right)=\xi(\xi+1) x_{1} x_{2}^{2}$. Since $\xi(\xi+1)$ is a unit of $R$, this implies that $M_{\phi}$ contains $x_{1} x_{2}^{2}$.

Now, we prove Theorem 2.1. Thanks to Lemmas 3.1 and 3.2 and (2.1), it suffices to show that $\Gamma:=\left\langle\operatorname{Aff}_{n+1}(R), \phi\right\rangle$ contains $\hat{\epsilon}(f)$ for each $f \in M_{\phi}$. Write $\phi=\left(f_{1}, \ldots, f_{n}\right)$ and

$f=h+\sum_{i=1}^{n} \sum_{j=1}^{r} a_{i, j} \eta_{j}\left(f_{i}\right)$, where $h \in \sum_{i=1}^{n} R x_{i}+R, a_{i, j} \in R$ and $\eta_{j} \in \operatorname{Aff}_{n}(R)$.

Then, $\hat{\epsilon}(f)$ is the product of $\hat{\epsilon}(h)$ and $\hat{\epsilon}\left(\eta_{j}\left(a_{i, j} f_{i}\right)\right)$ for $i=1, \ldots, n$ and $j=$ $1, \ldots, r$. Since $\Gamma$ contains $\hat{\epsilon}\left(f_{i}\right)$ for each $i$ by $(\mathrm{A}), \Gamma$ contains $\hat{\epsilon}\left(a_{i, j} f_{i}\right)$ for each $i, j$ by (B). Hence, $\Gamma$ contains $\eta_{j} \circ \hat{\epsilon}\left(a_{i, j} f_{i}\right) \circ \eta_{j}^{-1}=\hat{\epsilon}\left(\eta_{j}\left(a_{i, j} f_{i}\right)\right)$ for each $i, j$. Since $\hat{\epsilon}(h)$ is affine, it follows that $\Gamma$ contains $\hat{\epsilon}(f)$. This completes the proof of Theorem 2.1 .

\section{Proof of Theorem 2.2}

Assume that $p$ is zero or a prime. We define $\mathrm{NG}_{n}(R)$ to be the set of $\phi \in$ $\mathrm{GA}_{n}(R)$ such that no good monomial appears in $\phi\left(x_{1}\right), \ldots, \phi\left(x_{n}\right)$. If $p=0$, then we have $\mathrm{NG}_{n}(R)=\operatorname{Aff}_{n}(R)$. In this case, the following theorem is obvious. 
Theorem 4.1. $\mathrm{NG}_{n}(R)$ is a subgroup of $\mathrm{GA}_{n}(R)$, and no element of $\mathrm{NG}_{n}(R)$ is stably co-tame. In fact, $\mathrm{E}_{n}(R) \not \subset\left\langle\operatorname{Aff}_{n+r}(R), \mathrm{NG}_{n}(R)\right\rangle$ holds for any $r \geq 1$.

To prove Theorem 4.1, we need a lemma, where $p$ need not be zero or a prime. Consider the standard grading $R[\mathbf{x}]=\bigoplus_{l \geqslant 0} R[\mathbf{x}]_{l}$. An $R$-submodule $V$ of $R[\mathbf{x}]$ is said to be graded if $V=\bigoplus_{l \geqslant 0}\left(V \cap R[\mathbf{x}]_{l}\right)$. If $V$ is generated by monomials, then $V$ is graded. Recall that each $\left(f_{1}, \ldots, f_{n}\right) \in R[\mathbf{x}]^{n}$ is identified with the substitution map $R[\mathbf{x}] \rightarrow R[\mathbf{x}]$ defined by $x_{i} \mapsto f_{i}$ for $i=1, \ldots, n$, and $R[\mathbf{x}]^{n}$ forms a monoid for the composition defined in (1.1). Note that $V^{n}$ is closed under this operation if and only if $\phi(V) \subset V$ holds for each $\phi \in V^{n}$.

Lemma 4.2. Let $V$ be a graded $R$-submodule of $R[\mathbf{x}]$ such that $V^{n}$ is closed under composition. If $x_{1}, \ldots, x_{n} \in V$, then $V^{n} \cap \mathrm{GA}_{n}(R)$ is a subgroup of $\mathrm{GA}_{n}(R)$.

Proof. Since $V^{n} \cap \mathrm{GA}_{n}(R)$ contains $\left(x_{1}, \ldots, x_{n}\right)$, and is closed under composition, we show that $\phi^{-1}$ belongs to $V^{n}$ for each $\phi \in V^{n} \cap \operatorname{GA}_{n}(R)$. There exists $\eta \in V^{n} \cap \operatorname{Aff}_{n}(R)$ for which $\psi:=\phi \circ \eta$ satisfies $\psi\left(x_{i}\right) \in x_{i}+\bigoplus_{l \geqslant 2} R[\mathbf{x}]_{l}$ for $i=1, \ldots, n$. Since $\phi^{-1}=\eta \circ \psi^{-1}$, and $V^{n}$ is closed under composition, it suffices to verify that $\psi^{-1}$ belongs to $V^{n}$. Suppose to the contrary that $f:=\psi^{-1}\left(x_{i}\right)$ does not belong to $V$ for some $i$. Write $f=\sum_{l \geqslant 0} f_{l}$, where $f_{l} \in R[\mathbf{x}]_{l}$. Then, $f_{d} \notin V$ holds for some $d \geq 0$. Take the minimal $d$, and set $f^{\prime}:=\sum_{l<d} f_{l}$ and $f^{\prime \prime}:=\sum_{l>d} f_{l}$. Let $h, h^{\prime}$ and $h^{\prime \prime}$ be the homogeneous components of $\psi\left(f_{d}\right), \psi\left(f^{\prime}\right)$ and $\psi\left(f^{\prime \prime}\right)$ of degree $d$, respectively. Then, $h+h^{\prime}+h^{\prime \prime}$ belongs to $V$, since $\psi\left(f_{d}\right)+\psi\left(f^{\prime}\right)+\psi\left(f^{\prime \prime}\right)=\psi(f)=x_{i}$ belongs to $V$, and $V$ is graded by assumption. By the minimality of $d$, we have $f^{\prime} \in V$. Since $\psi=\phi \circ \eta$ belongs to $V^{n}$, it follows that $\psi\left(f^{\prime}\right)$ belongs to $V$. This implies that $h^{\prime}$ belongs to $V$ as before. As for $h$ and $h^{\prime \prime}$, we have $h=f_{d}$ and $h^{\prime \prime}=0$, since $\psi\left(x_{i}\right) \in x_{i}+\bigoplus_{l \geqslant 2} R[\mathbf{x}]_{l}$ holds for $i=1, \ldots, n$. Thus, $h+h^{\prime}+h^{\prime \prime}=f_{d}+h^{\prime}$ does not belong to $V$, a contradiction. Therefore, $\psi^{-1}$ belongs to $V^{n}$.

We remark that, if $V=\sum_{s \in \Sigma} A s$ for some $\Sigma \subset R[\mathbf{x}]$ and an $R$-subalgebra $A$ of $R[\mathbf{x}]$, and if $\phi(A) \subset A$ and $\phi(\Sigma) \subset V$ hold for each $\phi \in V^{n}$, then $V^{n}$ is closed under composition. For example, assume that $R$ has prime characteristic $p$, and define $R\left[\mathbf{x}^{p^{l}}\right]:=R\left[x_{1}^{p^{l}}, \ldots, x_{n}^{p^{l}}\right]$ for each $l \geq 0$. Set $\mathbf{Z}_{d}:=\{a \in \mathbf{Z} \mid a \geq d\}$ for each $d \in \mathbf{Z}$. Let $d, e \in \mathbf{Z}_{0}$ and $\emptyset \neq N \subset \mathbf{Z}_{0}$ be such that $d \leq e$, and each $u, v \in N$ satisfy $u+v \in N \cup \mathbf{Z}_{d}$. Then, we define a graded $R$-submodule of $R[\mathbf{x}]$ by

$$
V:=V(d, e, N):=R\left[\mathbf{x}^{p^{d}}\right]+\sum_{i=1}^{n} \sum_{u \in N} R\left[\mathbf{x}^{p^{e}}\right] x_{i}^{p^{u}} .
$$


Let us prove that $V^{n}$ is closed under composition using the remark for $A:=$ $R\left[\mathbf{x}^{p^{e}}\right]$ and $\Sigma:=R\left[\mathbf{x}^{p^{d}}\right] \cup\left\{x_{i}^{p^{u}} \mid 1 \leq i \leq n, u \in N\right\}$. First, note that $f \in V$ implies $f^{p^{v}} \in V$ for any $v \in N$, since each $u, v \in N$ satisfy $\left(x_{i}^{p^{u}}\right)^{p^{v}} \in\left\{x_{i}^{p^{w}} \mid\right.$ $w \in N\} \cup R\left[x_{i}^{p^{d}}\right]$, and $R\left[\mathbf{x}^{p^{d}}\right]$ contains $R\left[\mathbf{x}^{p^{e}}\right]$. Hence, $\phi\left(x_{i}^{p^{u}}\right)$ belongs to $V$ for each $\phi \in V^{n}, 1 \leq i \leq n$ and $u \in N$. Clearly, $\phi\left(R\left[\mathbf{x}^{p^{l}}\right]\right) \subset R\left[\mathbf{x}^{p^{l}}\right]$ holds for any $\phi \in R[\mathbf{x}]^{n}$ and $l \geq 0$. Therefore, we have $\phi(A) \subset A$ and $\phi(\Sigma) \subset V$ for each $\phi \in V^{n}$.

Now, let us prove Theorem 4.1 when $p$ is a prime. Clearly, $u, v \in\{0\}$ implies $u+v \in\{0\} \cup \mathbf{Z}_{1}$. So, we define $V_{n}:=V(1,1,\{0\})$ and $W_{n}:=$ $V(1,2,\{0\})$, i.e.,

$$
\begin{aligned}
V_{n} & =R\left[\mathbf{x}^{p}\right]+R\left[\mathbf{x}^{p}\right] x_{1}+\cdots+R\left[\mathbf{x}^{p}\right] x_{n} \\
W_{n} & =R\left[\mathbf{x}^{p}\right]+R\left[\mathbf{x}^{p^{2}}\right] x_{1}+\cdots+R\left[\mathbf{x}^{p^{2}}\right] x_{n} .
\end{aligned}
$$

If $n=p=2$, then $t_{1}, t_{2} \geq 0$ satisfy $x_{1}^{t_{1}} x_{2}^{t_{2}} \in W_{2}$ if and only if $t_{1}, t_{2} \equiv 0$ $(\bmod 2)$, or $t_{i} \equiv 1, t_{j} \equiv 0(\bmod 4)$ for some $i, j \in\{1,2\}$. Hence, we have $x_{1}^{t_{1}} x_{2}^{t_{2}} \notin W_{2}$ if and only if $x_{1}^{t_{1}} x_{2}^{t_{2}}$ is a good monomial. Similarly, when $(n, p) \neq$ $(2,2)$, a nonzero monomial $m$ is good if and only if $m$ does not belong to $V_{n}$. Thus, $\mathrm{NG}_{n}(R)$ is equal to $W_{2}^{2} \cap \mathrm{GA}_{2}(R)$ if $n=p=2$, and to $V_{n}^{n} \cap \mathrm{GA}_{n}(R)$ otherwise. Therefore, $\mathrm{NG}_{n}(R)$ is a subgroup of $\mathrm{GA}_{n}(R)$ by Lemma 4.2, Note that $\left\langle\operatorname{Aff}_{n+r}(R), \mathrm{NG}_{n}(R)\right\rangle$ is contained in $W_{n+r}^{n+r}$ if $n=p=2$, and in $V_{n+r}^{n+r}$ otherwise. Since $\mathrm{E}_{n}(R) \backslash V_{n+r}^{n+r}$ contains $\epsilon\left(x_{2} x_{3}\right)$ if $n \geq 3$, and $\epsilon\left(x_{2}^{2}\right)$ if $p \geq 3$, while $\mathrm{E}_{n}(R) \backslash W_{n+r}^{n+r}$ contains $\epsilon\left(x_{2}^{p+1}\right)$ for any $n, p \geq 2$, we get the last part of Theorem 4.1. This completes the proof.

If $I$ is a proper ideal of $R$, then each $\phi \in \mathrm{GA}_{n+r}(R)$ induces an element $\phi_{I}$ of $\operatorname{GA}_{n+r}(R / I)$. Since $\mathrm{E}_{n}(R) \ni \tau \mapsto \tau_{I} \in \mathrm{E}_{n}(R / I)$ is surjective, $(\sigma \circ \tau)_{I}=$ $\sigma_{I} \circ \tau_{I}$ for each $\sigma, \tau \in \operatorname{GA}_{n+r}(R)$, and $\tau \in \operatorname{Aff}_{n+r}(R)$ implies $\tau_{I} \in \operatorname{Aff}_{n+r}(R / I)$, we see that

$$
\mathrm{E}_{n}(R) \subset\left\langle\operatorname{Aff}_{n+r}(R), \phi\right\rangle \text { implies } \mathrm{E}_{n}(R / I) \subset\left\langle\operatorname{Aff}_{n+r}(R / I), \phi_{I}\right\rangle
$$

for any $r \geq 0$ and $\phi \in \mathrm{GA}_{n}(R)$. Hence, Theorem 4.1 implies the following corollary.

Corollary 4.3. Let $I$ be a proper ideal of $R$ such that $\operatorname{char}(R / I)$ is zero or a prime. If $\phi \in \mathrm{GA}_{n}(R)$ satisfies $\phi_{I} \in \mathrm{NG}_{n}(R / I)$, then $\mathrm{E}_{n}(R) \not \subset$ $\left\langle\operatorname{Aff}_{n+r}(R), \phi\right\rangle$ holds for any $r \geq 1$. Here, $\operatorname{char}(R / I)$ is the characteristic of $R / I$.

Now, we can prove Theorem 2.2. By assumption, $I:=I_{\phi}$ is a proper ideal of $R$. If $p$ is a prime, then $R$ contains $\mathbf{F}_{p}$, and so char $(R / I)=p$. Hence, $\phi_{I}$ belongs to $\mathrm{NG}_{n}(R / I)$ by the definition of $I$. Thus, $\phi$ is not stably co-tame 
by Corollary 4.3. If $p=0$, then $\phi_{I}$ is affine. By (4.1), this implies that $\phi$ is not stably co-tame.

Finally, we remark that, if $p=2$, then $R\left[x_{2}\right]$ is contained in $V_{n}$. Hence, we have $\mathrm{E}_{2}(R) \subset V_{2}^{2}$, and so $\mathrm{T}_{2}(R) \subset V_{2}^{2}$. Thus, for any $\phi \in \mathrm{T}_{2}(R)$, no monomial $x_{1}^{t_{1}} x_{2}^{t_{2}}$ with $t_{1}$ and $t_{2}$ odd appears in $\phi\left(x_{1}\right)$ and $\phi\left(x_{2}\right)$. If $R$ is a domain, the same holds for any $\phi \in \mathrm{GA}_{2}(R)$, since $\mathrm{T}_{2}(K)=\mathrm{GA}_{2}(K)$ for any field $K$ (cf. [J], [K]).

Lemma 4.4. Assume that $p=2, \phi \in \mathrm{GA}_{2}(R), 1 \leq i \leq 2$ and $t_{1}, t_{2} \geq 0$ are odd. If $a \in R$ is the coefficient of $x_{1}^{t_{1}} x_{2}^{t_{2}}$ in $\phi\left(x_{i}\right)$, then a belongs to the nilradical of $R$.

Proof. Let $\mathfrak{p}$ be any prime ideal of $R$. Since $R / \mathfrak{p}$ is a domain of characteristic two, $x_{1}^{t_{1}} x_{2}^{t_{2}}$ does not appear in $\phi_{\mathfrak{p}}\left(x_{i}\right)$ as mentioned. Hence, $a$ belongs to $\mathfrak{p}$.

\section{Proof of Theorem 2.3 (i)}

Assume that $R$ is a commutative $k$-algebra. To prove Theorem 2.3 (i), we verify that one of (a), (b) and (c) of Theorem 2.1 holds when $J_{\phi}=R$. If $J_{\phi}=R$, a good monomial appears in a polynomial satisfying the degree condition. This implies that $\# k>2$, and so there always exists $\xi \in R^{*}$ satisfying $\xi+1 \in R^{*}$.

Take any $f(x) \in R[x]$ and write $f(x)=\sum_{i=0}^{d} u_{i} x^{i p^{e}}$ with $d, e \geq 0$ and $u_{i} \in R$, where we regard $p^{e}=1$ if $p=0$. If $k$ contains $d+1$ distinct elements $\xi_{0}, \ldots, \xi_{d}$, then $u_{i} x^{i p^{e}}$ can be written as a $k$-linear combination of $f\left(\xi_{0} x\right), \ldots, f\left(\xi_{d} x\right)$ for each $0 \leq i \leq d$ by linear algebra. This remark is used to prove the following lemma.

Lemma 5.1. If $f \in R[\mathbf{x}]$ satisfies the degree condition, each monomial appearing in $f$ is written as a k-linear combination of $f\left(\xi_{1} x_{1}, \ldots, \xi_{n} x_{n}\right)$ for $\xi_{1}, \ldots, \xi_{n} \in k^{*}$.

Proof. We prove the lemma by induction on $n$. The case $n=0$ is clear. Assume that $n \geq 1$, and write $f=\sum_{i=0}^{d} f_{i} x_{1}^{i p^{e}}$, where $d:=\# k-2, f_{i} \in R[\hat{\mathbf{x}}]$ and $e \geq 0$. Take any monomial $m$ appearing in $f$. Then, there exists $i$ such that $m$ appears in $f_{i} x_{1}^{i}$. Since $\# k=d+2$, we may find distinct $\alpha_{0}, \ldots, \alpha_{d} \in k^{*}$. Then, $f_{i} x_{1}^{i}$ is written as a $k$-linear combination of $f\left(\alpha_{l} x_{1}, x_{2}, \ldots, x_{n}\right)$ for $l=0, \ldots, d$ as remarked. By the choice of $i$, we have $m=m^{\prime} x_{1}^{i}$ for some monomial $m^{\prime}$ appearing in $f_{i}$. Note that $\operatorname{deg}_{x_{j}}^{s} f_{i} \leq \operatorname{deg}_{x_{j}}^{s} f \leq d$ for all $j$. Hence, by induction assumption, $m^{\prime}$ is written as a $k$-linear combination of $f_{i}\left(\beta_{2} x_{2}, \ldots, \beta_{n} x_{n}\right)$ for $\beta_{2}, \ldots, \beta_{n} \in k^{*}$. Thus, $m=m^{\prime} x_{1}^{i}$ is written as a $k$-linear combination of $f_{i}\left(\beta_{2} x_{2}, \ldots, \beta_{n} x_{n}\right) x_{1}^{i}$ 's, and therefore that of $f\left(\alpha_{l} x_{1}, \beta_{2} x_{2}, \ldots, \beta_{n} x_{n}\right)$ for $l=0, \ldots, d$ and $\beta_{2}, \ldots, \beta_{n} \in k^{*}$. 
Next, we define $\lambda \in \operatorname{Aff}_{n}(R)$ by $\lambda\left(x_{i}\right)=x_{i}+1$ for $i=1, \ldots, n$.

Lemma 5.2. If $x_{1}^{t_{1}} \cdots x_{n}^{t_{n}}$ is a good monomial, there appears in $\lambda\left(x_{1}^{t_{1}} \cdots x_{n}^{t_{n}}\right)$ the monomial $u x_{i} x_{j}$ with $u \in k^{*}$ in the cases (I), (II) and (III), $x_{i} x_{j}^{2}$ in the case (IV), and $x_{i}^{3}$ in the case (V), where $i, j \in\{1, \ldots, n\}$, and where $i \neq j$ if (II) or (IV).

Proof. In the case (IV), we have $\lambda\left(x_{1}^{t_{1}} x_{2}^{t_{2}}\right)=\left(x_{i}+1\right)\left(x_{j}^{2}+1\right)\left(x_{i}^{4}+1\right)^{t_{i}^{\prime}}\left(x_{j}^{4}+1\right)^{t_{j}^{\prime}}$ for some $i, j \in\{1,2\}$ with $i \neq j$, and $t_{1}^{\prime}, t_{2}^{\prime} \geq 0$. Then, it is easy to see that the monomial $x_{i} x_{j}^{2}$ appears in $\lambda\left(x_{1}^{t_{1}} x_{2}^{t_{2}}\right)$. Other cases are verified similarly.

Now, let us prove Theorem 2.3 (i). Since $J_{\phi}=R$ by assumption, we may find $a_{1}, \ldots, a_{r} \in R$ and $g_{1}, \ldots, g_{r} \in \sum_{i=1}^{n} R \phi\left(x_{i}\right)+\sum_{i=1}^{n} R x_{i}$ with $r \geq 1$ as follows:

(1) For each $1 \leq l \leq r$, a good monomial $m_{l}$ appears in $g_{l}$ with coefficient $a_{l}$.

(2) $\sum_{l=1}^{r} a_{l}=1$, and $g_{1}, \ldots, g_{r}$ satisfy the degree condition.

Since $g_{l}$ satisfies the degree condition for each $l$, so does $m_{l}$, and hence so does $\lambda\left(m_{l}\right)$. Clearly, $g_{l}$ belongs to $M_{\phi}$. Hence, $m_{l}$ belongs to $M_{\phi}$ by Lemma 5.1 and (2.1). Then, $\lambda\left(m_{l}\right)$ belongs to $M_{\phi}$, so each monomial appearing in $\lambda\left(m_{l}\right)$ belongs to $M_{\phi}$ similarly. By Lemma 5.2, there appears in $\lambda\left(m_{l}\right)$ the monomial $n_{l}:=u_{l} a_{l} x_{i_{l}} x_{j_{l}}$ with $u_{l} \in k^{*}$ in the cases (I), (II) and (III), $n_{l}:=a_{l} x_{i_{l}} x_{j_{l}}^{2}$ in the case (IV), and $n_{l}:=a_{l} x_{i_{l}}^{3}$ in the case (V), where $i_{l}, j_{l} \in\{1, \ldots, n\}$, and where $i_{l} \neq j_{l}$ if (II) or (IV). Since $n_{l}$ belongs to $M_{\phi}$, we have $a_{l} x_{1} x_{2} \in M_{\phi}$ in the case (II). The proof of Lemma 3.2 shows that $a_{l} x_{1} x_{2}^{2} \in M_{\phi}$ in the case (V) as well as in the case (IV).

If $n \geq 3$ and $p=2$, then $m_{l}$ must be of type (II) for all $l$. Hence, $M_{\phi}$ contains $\sum_{l=1}^{r} a_{l} x_{1} x_{2}=x_{1} x_{2}$. Therefore, (a) of Theorem 2.1 holds.

If $n=p=2$, then $m_{l}$ is of type (II) or (IV) or (V) for each $l$. In the case (II), $a_{l}$ is nilpotent by Lemma 4.4. Since $\sum_{l=1}^{r} a_{l}=1$, it follows that $u:=\sum^{\prime} a_{l}$ is a unit of $R$, where the sum $\sum^{\prime}$ is taken over $l$ such that $m_{l}$ is of type $(\mathrm{IV})$ or $(\mathrm{V})$. Hence, $M_{\phi}$ contains $u^{-1} \sum^{\prime} a_{l} x_{1} x_{2}^{2}=x_{1} x_{2}^{2}$. Therefore, (c) of Theorem 2.1 holds.

If $p \neq 2$, then each $m_{l}$ is of type (I) or (II) or (III), so $M_{\phi}$ contains $u_{l} a_{l} x_{i_{l}} x_{j_{l}}$. By (2.1), $M_{\phi}$ contains $a_{l} x_{1}^{2}$ if $i_{l}=j_{l}$. If $i_{l} \neq j_{l}$, then $M_{\phi}$ contains $a_{l} x_{1} x_{2}$, and hence contains $a_{l} x_{1}\left(x_{2}+x_{1}\right)-a_{l} x_{1} x_{2}=a_{l} x_{1}^{2}$. Thus, $M_{\phi}$ contains $\sum_{l=1}^{r} a_{l} x_{1}^{2}=x_{1}^{2}$. Therefore, (b) of Theorem 2.1 holds. This completes the proof of Theorem 2.3 (i). 


\section{Remarks}

6.1. When $R=k$, Edo-Lewis [EL] showed that $\theta_{N}:=(\beta \circ \pi)^{-N} \circ \pi \circ(\beta \circ \pi)^{N}$ is neither affine nor co-tame for each $N \geq 3$, where

$$
\beta:=\left(x_{1}+x_{2}^{2}\left(x_{2}+x_{3}^{2}\right)^{2}, x_{2}+x_{3}^{2}, x_{3}\right) \quad \text { and } \quad \pi:=\left(x_{2}, x_{1}, x_{3}\right) .
$$

Here, we mention that the expression of $\theta_{N}$ above is slightly different from the original one, due to the difference in the definitions of composition (cf. $\S 2.2$ of [EL] and (1.1)). Since $R=k$ and $\theta_{N}$ is not affine, $\theta_{N}$ is stably co-tame if $p=0$ by the remark after Corollary 2.4. If $p=2$, then $\theta_{N}$ belongs to $\mathrm{NG}_{3}(k)$, and hence is not stably co-tame by Theorem 4.1 . When $p \geq 3$, we have the following theorem.

Theorem 6.1. If $p \geq 3, N \geq 1$ and $\# k \geq 4^{2 N-1}+2$, then $\theta_{N}$ is stably co-tame.

Proof. Observe that $\theta_{N}$ is written as $\sigma_{1} \circ \tau_{1} \circ \cdots \circ \sigma_{N} \circ \tau_{N} \circ \sigma$, where

$$
\sigma_{i} \in\left\{\pi \circ \beta \circ \pi, \pi \circ \beta^{-1} \circ \pi\right\}, \quad \tau_{i} \in\left\{\beta, \beta^{-1}\right\} \quad \text { and } \quad \sigma \in\{\mathrm{id}, \pi\}
$$

with $\sigma_{1}=\pi \circ \beta^{-1} \circ \pi=$ : $\left(h_{1}, h_{2}, x_{3}\right)$. For each $\mathbf{w}=\left(w_{1}, w_{2}, w_{3}\right) \in \mathbf{Z}^{3}$, we define the $\mathbf{w}$-weighted grading on $R[\mathbf{x}]$ by $\operatorname{deg}_{\mathbf{w}} x_{i}:=w_{i}$ for $i=1,2,3$. For $f \in R[\mathbf{x}] \backslash\{0\}$, we denote by $f^{\mathbf{w}}$ the highest $\mathbf{w}$-homogeneous part of $f$. Now, set $\theta_{N}^{\prime}:=\theta_{N} \circ \sigma$. We claim that, if $\operatorname{deg}_{\mathbf{w}} h_{2}$ is greater than $(1 / 4) \operatorname{deg}_{\mathbf{w}} h_{1}$ and $2 w_{3}$, then

$$
\theta_{N}^{\prime}\left(x_{i}\right)^{\mathbf{w}}=\left(\sigma_{1} \circ \tau_{1} \circ \cdots \circ \sigma_{N} \circ \tau_{N}\right)\left(x_{i}\right)^{\mathbf{w}}= \pm\left(h_{2}^{\mathbf{w}}\right)^{4^{2 N-i}} \text { for } i=1,2 .
$$

In fact, the case $N=1$ is checked directly, and the case $N \geq 2$ follows by induction on $N$, since $\left(\sigma_{i} \circ \tau_{i}\right)\left(x_{2}\right)=x_{2} \pm x_{1}^{2}\left(x_{1} \pm x_{3}^{2}\right)^{2} \pm x_{3}^{2}$ and $\left(\sigma_{i} \circ \tau_{i}\right)\left(x_{1}\right)=x_{1} \pm x_{3}^{2} \pm\left(x_{2} \pm x_{1}^{2}\left(x_{1} \pm x_{3}^{2}\right)^{2}\right)^{2}\left(x_{2} \pm x_{1}^{2}\left(x_{1} \pm x_{3}^{2}\right)^{2} \pm x_{3}^{2}\right)^{2} \quad$ for each $i$.

Since $h_{1}=x_{1}-x_{3}^{2}$ and $h_{2}=x_{2}-x_{1}^{2} h_{1}^{2}$, the assumption of the claim holds for $\mathbf{w}=(1,0,0),(0,1,0),(0,0,1),(2,0,1)$, for which

$$
h_{2}^{\mathbf{w}}=-x_{1}^{4}, x_{2},-x_{1}^{2} x_{3}^{4},-x_{1}^{2}\left(x_{1}-x_{3}^{2}\right)^{2},
$$

respectively. The first three of these imply $\operatorname{deg}_{x_{i}} \theta_{N}^{\prime}\left(x_{2}\right) \leq 4 \cdot 4^{2 N-2} \leq$ $\# k-2$ for $i=1,2,3$. Hence, $\theta_{N}^{\prime}\left(x_{2}\right)$ satisfies the degree condition. Since $\theta_{N}^{\prime}\left(x_{2}\right)^{(2,0,1)}= \pm\left(x_{1}\left(x_{1}-x_{3}^{2}\right)\right)^{t}$ with $t:=2 \cdot 4^{2 N-2}$ similarly, the monomial $\pm\left(x_{1} x_{3}^{2}\right)^{t}$ appears in $\theta_{N}^{\prime}\left(x_{2}\right)$. Since $p \geq 3$, we see that $x_{1}^{t} x_{3}^{2 t}$ is a good monomial of type (III). Thus, we get $J_{\theta_{N}}=J_{\theta_{N}^{\prime}}=R$. Therefore, $\theta_{N}$ is stably co-tame by Theorem 2.3 (i). 
6.2. When $k$ is a finite field, Theorem 2.3 might not be useful due to the degree condition. In such a case, the following technique may be effective. Take any $\eta \in \operatorname{Aff}_{n}(R)$, and set $\delta:=\eta-\mathrm{id}$. Then, $\delta\left(M_{\phi}\right) \subset M_{\phi}$ holds for each $\phi \in \mathrm{GA}_{n}(R)$ by (2.1). Since $\phi\left(x_{j}\right)$ belongs to $M_{\phi}$ for each $j$, we get the following lemma.

Lemma 6.2. If $\phi \in \mathrm{GA}_{n}(R)$ and $h \in R[\mathbf{x}]$ satisfy the following condition, then $h$ belongs to $M_{\phi}$ : There exist $1 \leq j \leq n$ and $\eta_{1}, \ldots, \eta_{l} \in \operatorname{Aff}_{n}(R)$ with $l \geq 1$ such that $\left(\delta_{1} \circ \cdots \circ \delta_{l} \circ \phi\right)\left(x_{j}\right)$ belongs to $R^{*} h+M_{\phi}$, where $\delta_{i}:=\eta_{i}-$ id for each $i$.

Assume that $p$ is a prime. For $i=1, \ldots, n$, take any $a_{i} \in R^{*}$, define $\eta_{i} \in \operatorname{Aff}_{n}(R)$ by $\eta_{i}\left(x_{i}\right)=x_{i}+a_{i}$ and $\eta_{i}\left(x_{j}\right)=x_{j}$ for $j \neq i$, and put $\delta_{i}:=$ $\eta_{i}-\mathrm{id}$. Then, we have $\delta_{i}^{p}=0$ since $\eta_{i}^{p}=\mathrm{id}, \operatorname{deg}_{x_{i}} \delta_{i}\left(x_{i}^{d}\right)<d$ for $d \geq 0$, and $\delta_{i}^{t}\left(x_{i}^{d}\right) \in R^{*} x_{i}^{d-t}+\sum_{j=0}^{d-t-1} R x_{i}^{j}$ if $p \nmid d-l$ for all $0 \leq l<t$. Note that $\operatorname{ker} \delta_{i}$ is an $R$-subalgebra of $R[\mathbf{x}]$, and $\delta_{i}$ is ker $\delta_{i}$-linear. Also, ker $\delta_{i}$ contains the $R$-subalgebra $A_{i}$ generated by $q_{i}:=x_{i}^{p}-a_{i}^{p-1} x_{i}$ and $x_{j}$ for $j \neq i$. We claim that $\operatorname{ker} \delta_{i}=A_{i}$. In fact, each $f \in \operatorname{ker} \delta_{i} \backslash\{0\}$ satisfies $p \mid \operatorname{deg}_{x_{i}} f=: l p$, hence $\operatorname{deg}_{x_{i}}\left(f-h q_{i}^{l}\right)<l p$ holds for some $h \in R\left[\left\{x_{j} \mid j \neq i\right\}\right]$. Thus, we get $f-h q_{i}^{l} \in A_{i}$ by induction on $l$, and so $f \in A_{i}$.

Now, fix $l=\left(l_{1}, \ldots, l_{n}\right) \in\{0, \ldots, p-1\}^{n}$, and set $\delta^{l}:=\delta_{1}^{l_{1}} \circ \cdots \circ \delta_{n}^{l_{n}}$ and

$$
M_{l}:=\sum_{i=0}^{n} R x_{i} \mathbf{x}^{l}+\sum_{i=1}^{n} \sum_{j=0}^{l_{i}-1} A_{i} x_{i}^{j}, \text { where } x_{0}:=1 \text { and } \mathbf{x}^{l}:=x_{1}^{l_{1}} \cdots x_{n}^{l_{n}} .
$$

Then, since $\delta_{i}$ 's commute with each other, and are $A_{i}$-linear, we see that $\delta^{l}\left(M_{l}\right)$ is contained in $\sum_{i=0}^{n} R x_{i}$. Hence, $\delta^{l}\left(M_{l}\right) \subset M_{\phi}$ holds for every $\phi \in$ $\mathrm{GA}_{n}(R)$. Using this, we can give sufficient conditions for stably co-tameness of $\phi$. For example, assume that $l_{1}, l_{2} \leq p-2$. Then, $\delta^{l}\left(x_{1} x_{2} \mathbf{x}^{l}\right)$ belongs to $R^{*} x_{1} x_{2}+\sum_{i=0}^{2} R x_{i}$. Hence, if $\phi$ satisfies $\phi\left(x_{j}\right) \in R^{*} x_{1} x_{2} \mathbf{x}^{l}+M_{l}$ for some $1 \leq j \leq n$, then $\left(\delta^{l} \circ \phi\right)\left(x_{j}\right)$ belongs to $R^{*} x_{1} x_{2}+M_{\phi}$. This implies that $x_{1} x_{2}$ belongs to $M_{\phi}$ by Lemma 6.2. Therefore, $\phi$ is stably co-tame by Theorem 2.1. Similarly, if $p \geq 3, l_{1} \leq p-3$, and $\phi\left(x_{j}\right)$ belongs to $R^{*} x_{1}^{2} \mathbf{x}^{l}+M_{l}$ for some $1 \leq j \leq n$, then $\phi$ is stably co-tame.

6.3. When $n \geq 3$, Motoki Kuroda $\mathrm{MK}$ showed that $\epsilon\left(x_{2}^{2}\right)$ is stably cotame for $R=\mathbf{F}_{p}$ with $p \geq 3$, and found it hard to decide whether $\epsilon\left(x_{2}^{5}\right)$ is stably co-tame for $R=\mathbf{F}_{3}$. Note that $\epsilon\left(x_{2}^{5}\right)$ is stably co-tame for $R=\mathbf{F}_{9}$ by Theorem 2.3 (ii).

6.4. The Tame Generators Problem asks if $\mathrm{GA}_{n}(R)=\mathrm{T}_{n}(R)$. In the context of stably co-tame automorphisms, $\mathrm{NG}_{n}(R)$ is a natural generalization of $\operatorname{Aff}_{n}(R)$. So, the following problem is of interest, as a generalization of the 
Tame Generators Problem in prime characteristic (see also [EK] for other generalizations).

Problem. When $p$ is a prime, does it hold that $\mathrm{GA}_{n}(R)=\left\langle\mathrm{NG}_{n}(R), \mathrm{T}_{n}(R)\right\rangle$ ?

\section{References}

[BEW] J. Berson, A. van den Essen and D. Wright, Stable tameness of two-dimensional polynomial automorphisms over a regular ring, Adv. Math. 230 (2012), no. 4-6, 2176-2197.

[B] Y. Bodnarchuk, On generators of the tame invertible polynomial maps group, Internat. J. Algebra Comput. 15 (2005), no. 5-6, 851-867.

[Ed] E. Edo, Coordinates of $R[x, y]$ : constructions and classifications, Comm. Algebra 41 (2013), no. 12, 4694-4710.

[EK] E. Edo and S. Kuroda, Generalisations of the tame automorphisms over a domain of positive characteristic, Transform. Groups 20 (2015), no. $1,65-81$.

[EL] E. Edo and D. Lewis, The affine automorphism group of $\mathbb{A}^{3}$ is not a maximal subgroup of the tame automorphism group, Michigan Math. J. 64 (2015), no. 3, 555-568.

[Es] A. van den Essen, Polynomial automorphisms and the Jacobian conjecture, Progress in Mathematics, Vol. 190, Birkhäuser, Basel, Boston, Berlin, 2000.

[J] H. Jung, Über ganze birationale Transformationen der Ebene, J. Reine Angew. Math. 184 (1942), 161-174.

[K] W. van der Kulk, On polynomial rings in two variables, Nieuw Arch. Wisk. (3) 1 (1953), 33-41.

[MK] M. Kuroda, Stably Derksen polynomial automorphisms over finite fields (Japanese), Master's Thesis, Tokyo Metropolitan University, January 2016.

[MW] S. Maubach and R. Willems, Polynomial automorphisms over finite fields: mimicking tame maps by the Derksen group, Serdica Math. J. 37 (2011), no. 4, 305-322 (2012). 
[N] M. Nagata, On Automorphism Group of $k[x, y]$, Lectures in Mathematics, Department of Mathematics, Kyoto University, Vol. 5, Kinokuniya Book-Store Co. Ltd., Tokyo, 1972.

[SU] I. Shestakov and U. Umirbaev, The tame and the wild automorphisms of polynomial rings in three variables, J. Amer. Math. Soc. 17 (2004), $197-227$.

[S] M. K. Smith, Stably tame automorphisms, J. Pure Appl. Algebra 58 (1989), 209-212.

Department of Mathematics and Information Sciences

Tokyo Metropolitan University

1-1 Minami-Osawa, Hachioji

Tokyo 192-0397, Japan

kuroda@tmu.ac.jp 